\title{
FACTORES ASOCIADOS AL ABANDONO DE LA ANTICONCEPCIÓN HORMONAL ORAL: UN ESTUDIO CON LOS ADOLESCENTES DE LA CIUDAD DE RIO DE J ANEIRO
}

\author{
Sandra de Morais Pereira ${ }^{1}$, Stella R. Taquette ${ }^{2}$ \\ ${ }^{1}$ Servicio de Ginecología, Hospital Geral de Bonsucesso, Universidad del Estado de Rio de Janeiro, Brazil. ${ }^{2}$ Facultad de \\ Ciencias Médicas, Universidad del Estado de Rio de Janeiro. NESA. Doctora en Medicina de la Universidad de São \\ Paulo/ Ribeirão Preto, Brazil.
}

\begin{abstract}
RESUMEN
Antecedentes: El anticonceptivo hormonal oral es el método más usado por las adolescentes. Objetivo: Teniendo como objetivo contribuir con la reducción de las tasas de embarazos no previstos y sus riesgos médicos y sociales, estudiamos los factores que llevan al abandono de esta técnica. Método: Estudio experimental no controlado, con el uso de anticonceptivo oral, por seis meses, en adolescentes de 15 a 19 años, que concurren al Hospital Geral de Bonsucesso (Rio de Janeiro, Brasil) desde noviembre de 2004 a agosto de 2005. Las participantes fueron entrevistadas y se sometieron a los exámenes clínicos y de laboratorio, antes y después del uso de la medicación. Resultados: En el análisis de los datos fue utilizada la prueba Chi cuadrado con nivel de significación menor al 5\%. Se constató que la edad precoz (15 a 16 años), la baja escolaridad y la relación conflictiva de la adolescente con la familia, son factores que están significativamente asociados a la no adhesión al método. Conclusiones: Para reducir la ocurrencia de embarazos no planeados son necesarios programas de salud reproductiva para adolescentes que incluyan la participación de sus familias, e inversiones que proporcionen el acceso universal y la valorización de la educación.
\end{abstract}

PALABRAS CLAVES: Adolescencia, anticoncepción, abandono, embarazo en la adolescencia

\section{SUMMARY}

Background: The oral hormonal contraceptive is the most adopted method among adolescents. Objective: Seeking to reduce the rates of undesired pregnancy and all the medical and social risks, we studied the causing factors of the abandonment of this method. Methods: A non-controlled experimental study of the use of oral contraceptive was accomplished, for six months, with adolescents from 15 to 19 years old attended in the Hospital Geral de Bonsucesso (Rio de Janeiro, Brazil) between November 2004 and August 20005. The participants were interviewed and submitted to clinical and laboratorial tests before and after using the medication. In the data analysis the chi-square test was used with $5 \%$ level of significance. Results: It was verified that the precocious age (of 15 to 16 years old), the low education and the adolescent's conflicting relationship with their family are significant factors to the non-adhesion to the contraceptive method in question. Conclusions: We concluded that in order to reduce the occurrence of undesired pregnancies it will be necessary the adoption of programs of reproductive health for adolescents which include the participation of their families, as well as investments to provide the universal access and the valorization of the education.

KEY WORDS: Adolescence, contraception, abandonment, pregnancy in adolescence 


\section{INTRODUCCION}

En la adolescencia la iniciación sexual se ha mostrado cada vez más precoz, lo que aumenta la ocurrencia de gestaciones (1). Conforme al Instituto Brasileño de Geografía y Estadística (IBGE), los índices generales de fecundidad en Brasil presentan una progresiva declinación, lo mismo no acontece cuando analizamos las mujeres de 15 a 19 años. Estos datos nos llevan a evaluar lo que representa una gestación en la adolescencia en el ámbito médico y sobre todo social (2).

Fonseca (3) describe el inicio de la "vida sexual" como un rito de paso de la infancia para la vida adulta. Este momento es de suma importancia para la joven, y puede dejar marcas imborrables para el resto de su vida cuando no está preparada y sin afecto. Según Taquette y cols (4), la iniciación sexual de la adolescente cuando ocurre en edades más precoces, frecuentemente está asociada a la presión de los compañeros y a la influencia del grupo de iguales, sin que la joven tenga seguridad y control sobre la situación. La desigualdad de poder en las relaciones entre hombres y mujeres es uno de los motivos de la dificultad que ambos tienen en discutir formas seguras de ejercer la sexualidad $(5,6)$. Magalhães $(7)$ en su estudio sobre el embarazo recurrente en la adolescencia, observa en cuanto a la anticoncepción, que todavía es enfrentada como una responsabilidad únicamente femenina.

Varios estudios realizados en diferentes ciudades brasileñas identifican el embarazo en la adolescencia como factor de riesgo para bajo peso al nacer, prematurez y mortalidad neonatal precoz. Además, algunos de estos estudios resaltan un menor número de consultas prenatales y una mayor tasa de mortalidad infantil cuando la madre es menor de 18 años (8-11).

Los anticonceptivos hormonales orales son la forma más popular de control de la fertilidad en el mundo, en particular por las adolescentes (12) y lo más empleado entre los métodos reversibles en Europa y Estados Unidos (13). Además, son métodos eficientes, de buena reversibilidad, con poco o ningún efecto colateral (14).

El Hospital Geral de Bonsucesso, lugar de trabajo de una de las autoras, situado en la Zona Norte de la ciudad de Rio de Janeiro, región densamente poblada, dispone de un Policlínico de ginecología de adolescentes. Hemos observado un aumento de embarazos de riesgo que resultan muchas veces en pérdidas fetales, prematurez y enfermedades maternas. Debido a esto, este estu- dio fue delineado, con el objetivo de conocer los factores que llevan a las adolescentes al abandono de la anticoncepción hormonal oral, buscando colaborar con la reducción de las tasas de embarazo no planeado y los riesgos médicos y sociales.

\section{POBLACIÓN ESTUDIADA Y MÉTODO}

Estudio de corte prospectivo, cuya población blanco está compuesta por pacientes provenientes del Policlínico de Ginecología del Hospital Geral de Bonsucesso (HGB) - Sector de Adolescentes, que espontáneamente asistieron buscando orientación anticonceptiva, en el período noviembre de 2004 y agosto de 2005. La muestra estudiada fue de conveniencia y seleccionada entre las pacientes que buscaron servicio ginecológico y optaron por el método. Inicialmente, las adolescentes participaron de reuniones sobre "Sexualidad y Anticoncepción", realizadas mensualmente con las médicas ginecólogas y pediatras, profesionales del área de enfermería, servicio social y psicología. Enseguida, recibieron la orientación necesaria para la elección del método anticonceptivo, además del énfasis sobre la necesidad de la dupla protección (un anticonceptivo eficaz, asociado al preservativo). Después esta actividad fueron dirigidas para servicio ginecológico, clínico $y$, cuando fue necesario, a los demás profesionales del equipo. Aquéllas que después de la reunión escogieron el anticonceptivo hormonal oral fueron invitadas a participar del estudio.

Se observaron como criterios de inclusión: edad de 15 a 19 años, vida sexual activa, ausencia de uso previo de anticonceptivos hormonales, deseo de utilización del método hormonal oral de forma continuada y disposición para cumplir las exigencias de la pesquisa. Además, la evaluación clínica y de laboratorio solo incluyó aquéllas que no eran portadoras de enfermedades crónicas previas. Se excluyeron las pacientes que presentaban dificultades en cumplir los requisitos del estudio, tuviesen sospecha o embarazo confirmado, y fuesen portadoras de algunas contraindicaciones relativas o absolutas al uso de terapia hormonal, validadas por la FEBRASGO (15).

El anticonceptivo utilizado fue de baja dosificación, conteniendo $20 \mathrm{mcg}$ de etinil estradiol y 150 $\mathrm{mcg}$ de desogestrel, siendo un medicamento ya testado y de uso comercial. El anticonceptivo hormonal oral fue ofrecido por 6 meses y después del tercer ciclo fueron evaluados los síntomas y rehechas las orientaciones. Las pacientes fueron so- 
metidas a la anamnesis, examen físico y ginecológico completo, incluyendo examen colpocitológico. Todos esos parámetros fueron revistos después del sexto ciclo de tratamiento, cerrándose el estudio. La consulta incluyó la recogida de datos personales como edad, escolaridad, utilización de preservativo e informaciones sobre participación de la familia y su conocimiento sobre la actividad sexual de la adolescente.

Todas las adolescentes iniciaron la medicación en el primer día del ciclo menstrual, utilizando una pastilla por día durante 21 días consecutivos, seguidos por 7 días de intervalo y hasta que fuesen completados 6 ciclos. Las jóvenes fueron debidamente orientadas sobre las consecuencias posibles durante el uso de la medicación. Las consultas de seguimiento fueron realizadas después del 3ํㅡ y 6o ciclos de tratamiento. Sin embargo, durante todo el estudio nos colocamos a la disposición de las pacientes en caso de dudas. Las integrantes de la investigación pudieron contar también con la cooperación de las profesionales de asistencia social y psicología con servicio individualizado.

Para análisis de los datos fueron creadas categorías relacionadas al conocimiento e interés de la familia en la sexualidad de las jóvenes. Consideramos separadamente la participación del padre, de la madre, de ambos o de otros responsables en el proceso de anticoncepción de la adolescente y el apoyo recibido para que si sintiese segura y pudiese dar continuidad a sus proyectos. El uso de preservativos también fue objeto de análisis. En cuanto a la escolaridad, utilizamos la variable retraso escolar cuando había un desfase mayor que dos años con relación a la edad esperada para la serie frecuentada.

Fue definido como abandono, la interrupción del tratamiento, por cualesquier motivo, o la no comparecencia a la consulta de seguimiento después del tercer ciclo. Se efectuó búsqueda activa de las adolescentes que se ausentaron para lograr la confirmación del abandono y su justificación.

Para el procesamiento de los datos fue creado, en el programa SPSS versión 8.0, un banco de datos, para inserción de los cuestionarios de las adolescentes sometidas a la investigación y análisis estadístico. En la fase de computación de los datos, se realizó la descripción de frecuencias simples, el cruce de variables y algunos tests para verificación de significación estadística. Fue hecho el análisis univariado de todas las variables del estudio. Todas las observaciones fueron cruzadas según la variable que se refiere al abandono del tratamiento. En el transcurrir del texto, esas diferencias sólo son mencionadas cuando son estadísticamente significativas $(p<0,05)$ según Chi cuadrado de Pearson.

El protocolo de investigación fue aprobado por el comité de ética de la Universidad del Estado de Rio de Janeiro (UERJ), y cumplió los principios éticos contenidos en la declaración de Helsinki, y en la Resolución 196/96 del Consejo Nacional de Salud.

\section{RESULTADOS}

Fueron estudiadas 109 adolescentes con edades entre 15 y 19 años y media de 16,8 años. El inicio de la actividad sexual ocurrió con mayor incidencia a los 15 años. En el análisis de la escolaridad verificamos que: $27,5 \%$ poseían el $1^{\circ}$ Grado incompleto; 2,8\% el 1ํ Grado completo; cursaban el $2^{\circ}$ Grado 54,1\%; habían completado la Enseñanza Media 9,2\%; y, solo 6,4\% jóvenes hacían el $3^{\circ}$ Grado. El retraso escolar fue observado en un $33,9 \%$ de las participantes.

En la evaluación sobre el apoyo familiar recibido por las pacientes $22,9 \%$ informaron que solamente la madre era participativa, $43,2 \%$ ambos progenitores, $4,6 \%$ otros responsables y 29,3 no tuvieron cualquier tipo de apoyo por parte de la familia. De las pacientes que iniciaron el estudio, $74 \%$ permanecieron y $26 \%$ abandonaron por motivos diversos (Figura 1).

En el universo de adolescentes que abandonaron el método, 32,1\% informaron el uso incorrecto del anticonceptivo, siendo el "olvido" una constante. Cuando cuestionadas mostraban desinterés y falta de motivación en dar continuidad al tratamiento. El uso del anticonceptivo oral exige regu-

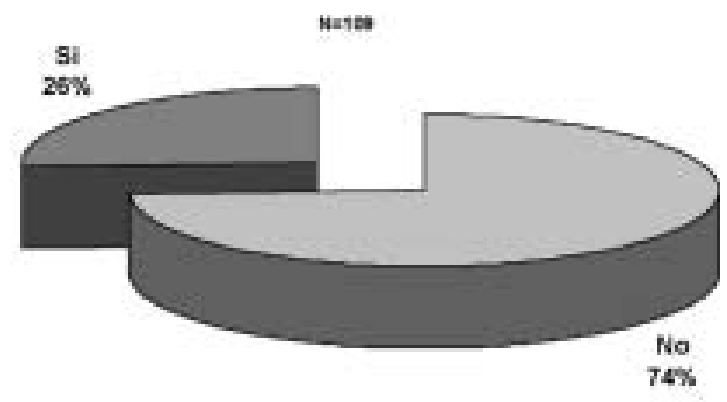

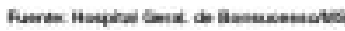

Figura 1. Distribución proporcional de las adolescentes del HGB estudiadas en la investigación conforme al abandono del método anticonceptivo, Río de Janeiro 2004-2005. 
laridad, y no es raro las adolescentes olviden de tomar la medicina. Del total de jóvenes que abandonaron el tratamiento, $17,9 \%$ declararon que no estaban con compañero, por eso interrumpieron el estudio.

El cambio de domicilio hizo que el $14,3 \%$ de las participantes abandonen la pesquisa, debido a la transferencia de lugares distantes del Hospital Geral de Bonsucesso, impidiéndolas de dar continuidad al tratamiento.

La necesidad de trabajar fue el motivo presentado para el abandono del estudio por $10,7 \%$ de las integrantes del universo de las desistentes. Según las mismas, trabajar dificultaba la comparecencia a las consultas regulares y al retiro de los anticonceptivos.

Sólo una de las jóvenes presentó náuseas y vómitos con el uso del anticonceptivo que la desmotivó a dar continuidad al tratamiento. El único abandono por este motivo fue, probablemente, consecuencia de la orientación recibida sobre el método y sus posibles efectos colaterales. Abandonaron sin causas determinadas $17,9 \%$ de las pacientes. Una joven de 15 años refirió "sentir pereza" de comparecer al hospital y cumplir los requisitos del estudio, otra alegó necesidad de interrumpir el tratamiento pues iniciaría un retiro espiritual y $10,7 \%$ no justificaron la interrupción.

Durante el estudio 10,7\% embarazaron, 7,1\% por el uso incorrecto ("olvido") del anticonceptivo, y $3,6 \%$ porque después del tercer mes de tratamiento deseó suspenderlo para embarazar.

Las causas de abandono se presentan detalladamente en la Tabla I.

Durante la investigación fue observado que las jóvenes de edad entre 15 y 16 años, tuvieron la mayor incidencia de no adhesión del anticonceptivo, aunque fuesen hechos diversos esfuerzos para la continuidad del estudio (Tabla II), mientras la edad de la primera relación sexual no fue significativa.

Evaluando la escolaridad del universo estudiado se identifica que entre las participantes del estudio que presentaban retraso escolar, 45,9\% abandonaron el anticonceptivo, este porcentaje muy superior al registrado en el conjunto de aquellas que no presentaban retraso (Figura 2).

Analizando los obstáculos existentes para el uso consistente del anticonceptivo, se identificó diferencia significativa entre el abandono del método y el retraso escolar.

Otro dato observado durante este estudio fue el elevado porcentaje de pacientes $(70,6 \%$, correspondiendo a 70 adolescentes) que dijeron no
Tabla I

DISTRIBUCIÓN ABSOLUTA Y PROPORCIONAL DE LOS MOTIVOS DE ABANDONO DEL USO DE LA ANTICONCEPCIÓN HORMONAL ORAL POR LAS ADOLESCENTES ESTUDIADAS

\begin{tabular}{lcr}
\hline Motivo de abandono & $n$ & $\%$ \\
\hline Deseo de embarazo & 1 & 3,6 \\
Uso incorrecto & 9 & 32,1 \\
Sin pareja & 5 & 17,9 \\
Cambio de dirección & 4 & 14,3 \\
Trabajo & 3 & 10,7 \\
Náuseas y vómitos & 1 & 3,6 \\
Otros & 5 & 17,9 \\
\hline Total & 28 & 100 \\
\hline
\end{tabular}

Fuente: Hospital Geral de Bonsucesso/ MS.

usar, o usar de forma irregular el preservativo, mientras solamente 32 jóvenes $(29,4 \%)$ lo usaban regularmente. Los factores como edad o escolaridad no se mostraron significativos.

\section{DISCUSIÓN}

Durante el estudio las 109 adolescentes se mostraron motivadas con el método anticonceptivo escogido, presentaron interés en informaciones, hablaron de sus proyectos y de la preocupación con su salud física y sexual. Hubo participación activa de muchachos en las reuniones sobre sexualidad y anticoncepción, qué nos lleva a creer

\section{Tabla II}

DISTRIBUCIÓN ABSOLUTA Y PROPORCIONAL ENTRE EDAD CRONOLÓGICA Y ABANDONO DEL USO DEL ANTICONCEPTIVO HORMONAL ORAL POR LAS ADOLESCENTES ESTUDIADAS

\begin{tabular}{lcccrrrr}
\hline Edad & \multicolumn{3}{c}{ Si } & \multicolumn{2}{c}{ Abandono } & \multicolumn{2}{c}{ Total } \\
& $n$ & $\%$ & $n$ & $\%$ & $n$ & $\%$ \\
\hline 15 & 9 & 32,1 & 12 & 14,8 & 21 & 19,3 \\
16 & 9 & 32,1 & 17 & 21,0 & 26 & 23,9 \\
17 & 4 & 14,3 & 26 & 32,1 & 30 & 27,5 \\
18 & 2 & 7,1 & 16 & 19,8 & 18 & 16,5 \\
19 & 4 & 14,3 & 10 & 12,3 & 14 & 12,8 \\
\hline Total & 28 & 100,0 & 81 & 100,0 & 109 & 100,0 \\
$\%$ & 25,7 & & 74,3 & & 100,0 & \\
\hline
\end{tabular}

Fuente: Hospital Geral de Bonsucesso/ MS

Fue identificada una diferencia estadísticamente significativa para el nivel del $5 \%$. 


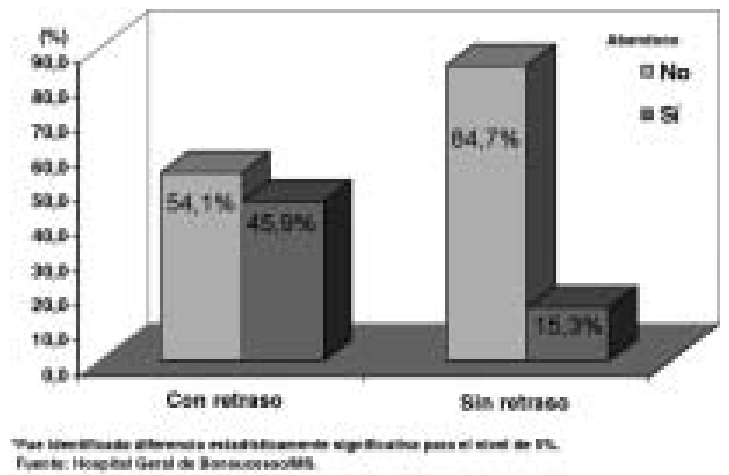

Figura 2. Distribución proporcional entre la información del atraso escolar y el abandono del uso de la anticoncepción hormonal oral en las adolescentes estudiadas en el HGB, RJ, 2004-2005.

que el tema abordado y el enfoque dado fueron adecuados y oportunos. Lo mismo para las 28 pacientes que abandonaron la pesquisa, creemos que fueron beneficiosas las informaciones ofrecidas sobre sexualidad, anticoncepción y la necesidad de cuidado con su salud. A esto se sumaron también nociones de ciudadanía, por la cuales se buscó que adquiriesen conciencia del que pueden y deben exigir un tratamiento de calidad, y hacer sus opciones libres de cualquier preconcepto.

El uso no frecuente de preservativos, durante el estudio, nos revela que no hubo cambio de comportamiento entre los adolescentes, siendo que la mayoría continúa no utilizando este método. Hecho semejante es apuntado por Passos y cols (16) quienes observaron que es necesario ser revisado en vista de la necesidad de prevención de enfermedades de transmisión sexual. Mientras Almeida y cols (17) observan una inclinación de las jóvenes a la flexibilización del uso del preservativo masculino cuando la relación se vuelve estable. Greydanus y cols (18) afirman que los riesgos de los anticonceptivos son menores que los de un embarazo no deseado, y que el uso concomitante de los preservativos debe ser firmemente alentado entre los adolescentes.

Se constató que la edad precoz, la baja escolaridad y la relación conflictiva de la adolescente con la familia hacen significativo el abandono del método.

Según Campos y Reis (19), el inicio de la vida sexual es muy precoz y frecuentemente "clandestino". Las niñas, generalmente, comienzan la relación sexual sin consejos previos y llevan cerca de 12 a 18 meses para buscar una orientación médica adecuada.
Según Kunde y cols (20), para una buena elección del anticonceptivo es necesario el conocimiento de diferentes métodos, comparando sus índices de eficacia y continuidad de uso. Este último parámetro es muy importante, pues un método anticonceptivo demanda un período más o menos prolongado de uso, siendo la adhesión normalmente condicionada, entre otros factores, por la mayor facilidad de continuidad proporcionada. Rosenberg (21), refiere que el riesgo de abandono de la usuaria que presenta náuseas o sangrado inesperado es dos veces mayor que aquélla que no refiere uno de los síntomas.

En este estudio se observó que la mayor escolaridad estaba asociada a la mayor y más precoz adhesión a la anticoncepción. Igualmente, se constató que jóvenes con 5 o más años de escolaridad son menos probables de tener la primera relación sexual en la adolescencia; más propensas a usar anticonceptivos y presentan menos riesgos de embarazo, cuando son comparadas con aquéllas con menos de 5 años de escolaridad (22).

Taquette (23) realiza su investigación con adolescentes de clases trabajadoras, que antes del embarazo varias de estas ya habían abandonado la escuela. Según la autora, la dificultad de realización de esas jóvenes a través de la escuela las deja más vulnerables a la realización personal por la maternidad. Mientras que, por la visión de la ciencia, tener hijos temprano es perjudicial a la salud de las madres y de los bebés, para ellas es la oportunidad de realizar algún sueño en la vida. Por eso, aspiran a los papeles femeninos más tradicionales: ser esposas y madres.

Se notó durante este estudio que el abandono del método escogido fue menor por las pacientes cuyos responsables tenían conciencia de su vida sexual activa. La calidad familiar es importante fuente de referencia para las adolescentes. Gran parte de aquéllas que resolvieron embarazar temprano también tenían madres que experimentaron la maternidad en una edad precoz. La ascensión social de esas jóvenes en su medio, por la maternidad, también es un factor de importancia, dejando el papel de hija, pasando al de madre.

Saito (24) observa la responsabilidad de la familia, dentro del proceso de educación sexual, por ser el primer grupo educador de los valores y de la cultura. Añade, además, como determinantes de riesgo: las familias desestructuradas y negligentes, y la falta de límites y de amor, que no favorecen el desarrollo de la autoestima, elemento tan importante de protección. Se observa que la mejor adhesión a la anticoncepción, y en la prác- 
tica ocurre, cuando las jóvenes cuentan con el auxilio de los compañeros o responsable (25).

Oliveira Júnior (26) en su estudio del perfil de la adolescente embarazada en cuanto a los aspectos socio-demográficos, psicológicos y de salud mental y reproductiva, revela que la baja escolaridad, el abandono de la escuela y del trabajo, y la iniciación sexual precoz, presentan un riesgo social, económico y psicológico y, también, un riesgo para la salud sexual y reproductiva. Muchas veces el embarazo en la adolescencia pasa a ser un proyecto de las jóvenes, una forma de transición para la vida adulta. Dadoorian (27) se refiere a que el embarazo en la banda de edad en estudio es frecuentemente deseada, teniendo un significado individual y social. En las clases sociales menos favorecidas la maternidad les confiere un nuevo estatus social, el de ser mujer.

Hogan y cols (28) observaron que la estructura familiar influye directamente en el comportamiento sexual de la adolescente. En familias en las cuales los padres biológicos crían sus hijas, el inicio de la actividad sexual acontece más tarde del que en aquellas familias en las que solamente la madre, $u$ otro adulto que no es el padre, ejecuta la crianza.

Según Costa y cols (29) la negligencia y poco uso de la anticoncepción están relacionados a las características propias de la adolescencia, que además de la sensación de omnipotencia, tiene dificultad en asumir la actividad sexual negando, por tanto, la posibilidad de ocurrencia de embarazo. Mientras, otros factores como la precariedad de programas dirigidos a la salud reproductiva en esta banda de edad, además de la falta de orientación de la familia y de la escuela, constituyen agravantes para la ocurrencia de gestación precoz entre adolescentes.

En el abordaje en cuanto a la sexualidad y anticoncepción, sería ideal una orientación amplia por parte de la familia y de la escuela, eso, sin embargo, no suele ocurrir en nuestro medio. La falta de preparación de los padres y la inadecuación del sistema educacional dejan las jóvenes casi totalmente sin orientación, a merced de informaciones distorsionadas e incompletas. Con esas limitaciones, la responsabilidad recae sobre el médico. El papel del profesional de la salud debe ser el que ayuda en el sentido amplio y no solamente lo de repasar enseñanzas técnicas, que a pesar de ser necesarias, se muestran insuficientes delante de la carencia de información de los adolescentes. Pensando así, es necesario ofrecer orientación lo más clara posible, con el objetivo de explicar todas las implicaciones positivas y negativas de la experiencia sexual en esta banda de edad, dejando la decisión por cuenta de la joven.

Varios autores resaltan la necesidad de la asistencia a la joven por un equipo multiprofesional, que le ofrezca soporte informativo, psicológico, emocional, y que en la calidad del programa de planificación familiar los factores para reducir la no adhesión a los anticonceptivos escogidos son: a libre elección de los métodos y la información suministrada a los pacientes, además de la capacidad técnica del equipo y de la relación humana con las usuarias. Las acciones destinadas a los jóvenes deben priorizar las cuestiones de sexualidad sin el discurso único sobre el riesgo. Hay necesidad de implementación de servicios de guía y asistencia que sean dirigidos específicamente a este subgrupo de la población (30-32).

Se puede concluir que para la reducción en la ocurrencia de gestaciones no planeadas son necesarios programas de salud reproductiva para adolescentes que incluyan la participación de sus familias e inversiones que proporcionen el acceso universal y la valorización de la educación. Se buscó en este estudio ofrecer a las adolescentes un servicio adecuado, respetando los parámetros de calidad y creando un tratamiento exclusivo para esa banda de edad.

Los datos presentados se refieren a grupos específicos de adolescentes y no pueden ser generalizados para toda la población adolescente de Rio de Janeiro. Los resultados auxilian al entendimiento de las causas de abandono del método anticonceptivo hormonal oral y como intentar evitarlo, además de reforzar los datos de otras pesquisas citadas durante el artículo.

\section{BIBLIOGRAFÍA}

1. Monteiro DLM, Pires ES, Schenttini GA, Cunha AA. Perfil socioeconómico da adolescente e adesão à contracepção. In: Monteiro DLM, Cunha AA, Bastos AC. Gravidez na adolescência. Rio de Janeiro: Revinter; 1998;171-85.

2. IBGE. Instituto Brasileiro de Geografia e Estatística. Nupcialidade fecundidade 2000. http:// www.ibge.gov.br/nupcialidade fecundidade 2000 (19/ Set/2005).

3. Fonseca APF. Sexualidade. In: Coutinho MFG, Barros RR. Adolescência: uma abordagem prática. São Paulo: Atheneu; 2001;231-36.

4. Taquette SR, Vilhena MM, Paula MC. Doenças sexualmente transmissíveis e gênero: um estudo transversal entre adolescentes no Rio de Janeiro. 
Cad Saúde Pública 2004;20:282-90.

5. Mota MP. Gênero e sexualidade: fragmentos de identidade masculina nos tempos de AIDS. Cad Saúde Pública 1998;14:145-55.

6. Villela WV, Barbosa RM. Repensando as relações entre gênero e sexualidade. In: Parker R, Barbosa $\mathrm{RM}$, organizadores. Sexualidades brasileiras. Rio de Janeiro: Relume Dumará; 1996;189-99.

7. Magalhães RR. A gravidez recorrente na adolescência: o caso de uma Maternidade [Dissertação de Mestrado]. Rio de Janeiro: Instituto Fernandes Figueira, Fundação Oswaldo Cruz; 2001.

8. Gama SGN, Szwacwald CL, Leal MDC, Theme Filha MM. Gravidez na adolescência como fator de risco para baixo peso ao nascer no Município do Rio de Janeiro, 1996 a 1998. Rev Saúde Pública 2001;35: 74-80.

9. Andrade CLT, Szwacwald CL. Análise espacial da mortalidade neonatal precoce no Município do Rio de Janeiro, 1995-1996. Cad Saúde Pública 2001;17: 1199-210.

10. Simões VMF, Silva AAM, Bettiol H, Lamy-Filho $F$, Tonial SR, Mochel EG. Características da gravidez na adolescência em São Luís, Maranhão. Rev Saúde Pública 2003;37:559-65.

11. Goldenberg P, Figueiredo MCT, Silva RS. Gravidez na adolescência: pré-natal e resultados perinatais em Montes Claros, Minas Gerais, Brasil. Cad Saúde Pública 2005;21:1077-86.

12. Ramos L, Lopes G, editores. Gravidez na adolescência. In: Saúde da adolescente: manual de orientação. São Paulo: Febrasgo; 2001;58-78.

13. Zite NB, Shulman LP. New options in contraception for teenagers. Curr Opin Obstet Gynecol 2003;15: 385-9.

14. Halbe HW, Melo NR. Anticoncepção hormonal. In: Giordano MG. Ginecologia endócrina e da reprodução. São Paulo: Fundo Editorial BYK; 1999;161-79.

15. Aldrighi JM, Petta CA, editores. Anticoncepcionais orais combinados. In: Anticoncepção: manual de orientação. São Paulo: Ponto; 2004;16-33.

16. Passos MRL, Dias APV, Santos G, Ferreira DC, Arze WNC. Estudo comparativo: perfil dos adolescentes atendidos numa clínica de DST nos anos de 1995 a 2003. Adolescência \& Saúde 2005;2:15-24.

17. Almeida MCC, Aquino EML, Gaffikin L, Magnani R J. Uso da contracepção por adolescentes de escolas públicas na Bahia. Rev Saúde Pública 2003;37:56675.

18. Greydanus DE, Patel DR, Rimsza ME. Contraception in the adolescent: an update. Pediatrics 2001;107:562-73.

19. Campos SGV, Reis JTL. Gravidez na adolescência. In: Magalhães MLC, Reis JTL. Compêndio de Ginecologia Infanto-Juvenil. Rio de Janeiro: Medsi;
2003;305-315.

20. Kunde A, Freitas F, Passos EP, Cunha Filho JSL. Anticoncepção. In: Freitas F, Menke $\mathrm{CH}$, Rivoire W, Passos E. P. Rotinas em Ginecologia. Porto Alegre: Atmed, 2001;191-210.

21. Rosenberg MJ, Waugh MS, Meehan TE. Use and misuse of oral contraceptives: risk indicators for poor pill taking and discontinuation. Contraception 1995; 51(5):283-8.

22. Leite IC, Rodrigues RN, Fonseca MC. Fatores associados com o comportamento sexual e reprodutivo entre adolescentes das regiões Sudeste e Nordeste do Brail. Cad Saúde Pública 2004;20:474-81.

23. Taquette SR. Iniciação sexual da adolescente: 0 desejo, o afeto e as normas sociais [Tese de Doutorado]. São Paulo: Faculdade de Medicina de Ribeirão Preto, Universidade de São Paulo; 1997.

24. Saito MI. Visão histórica da sexualidade: reflexões e desafios. In: Saito MI, Silva LEV. Adolescência: prevenção e risco. São Paulo: Atheneu; 2001;121-7.

25. Leal MM, Amado CR. Anticoncepção na adolecência. In: Françoso LA, Gejer D, Reato LFN. Sexualidade e saúde reprodutiva na adolescência. São Paulo: Ateneu; 2001: 85-110.

26. Oliveira JR, FC. Perfil da adolescente grávida quanto aos aspectos sócio-demográficos, psicológicos e de saúde sexual e reprodutiva [Dissertação de Mestrado]. Curitiba: Universidade Federal do Paraná; 2003.

27. Dadoorian D. A gravidez desejada em adolescents de classes populares [Dissertação de Mestrado]. Rio de Janeiro: Pontifícia Universidade Católica do Rio de Janeiro; 1994.

28. Hogan DP, Sun RMA, Cornwell G. Sexual and fertility behaviors of american females aged 15-19 years: 1985, 1990 and 1995. Am J Public Health 2000; 90:1421-5.

29. Costa COM, Queiroz SS, Santos CAT, Barboni AR, Neto AFO, Rocha G, et al. Condições de gestação, parto e nascimento em adolescentes e adultas jovens: Santa Casa. Adolesc Latinoam 1999;1:24251.

30. Monteiro DLM, Cunha AA. Perfil reprodutivo da adolescente. J Bras Ginecol 1994;104:59-62.

31. Halbe HW, Díaz J. Qualidade de atenção no planejamento familiar: marco conceitual e situação no Brasil. In: Halbe HW. Tratado de Ginecologia. São Paulo: Roca; 2000;914-23.

32. Miranda AE, Gadelha AMJ, Szwarcwald CL. Padrão de comportamento relacionado às práticas sexuais e ao uso de drogas de adolescentes do sexo feminino residentes em Vitória, Espírito Santo, Brasil. Cad Saúde Pública 2005;21:207-16. 